Received

11 December 2014

Accepted

26 February 2015

Published

19 March 2015

\section{Correspondence and} requests for materials should be addressed to

J.K. (joonkim@ incheon.ac.kr) or D.-W.K. (dwkim@ ewha.ac.kr) \title{
nanocones for selective emitters: optical and electrical improvements of Si solar cells
}

Joondong Kim 1 , Ju-Hyung Yun' , Hyunyub Kim², Yunae Cho ${ }^{3}$, Hyeong-Ho Park' ${ }^{4}$, M. Melvin David Kumar', Junsin $\mathrm{Yi}^{2}$, Wayne A. Anderson ${ }^{5}$ \& Dong-Wook Kim ${ }^{3}$

\begin{abstract}
'Department of Electrical Engineering, Incheon National University, Incheon 406772, Korea, ${ }^{2}$ College of Information and Communication Engineering, Sungkyunkwan University, Suwon 440746, Korea, ${ }^{3}$ Department of Physics, Ewha Womans University, Seoul 120750, Korea, ${ }^{4}$ Applied Device and Material Lab., Device Technology Division, Korea Advanced Nanofab Center (KANC), Suwon 443270, Korea, ${ }^{5}$ Department of Electrical Engineering, University at Buffalo, State University of New York, Buffalo, New York 14260, USA.
\end{abstract}

Periodical nanocone-arrays were employed in an emitter region for high efficient Si solar cells. Conventional wet-etching process was performed to form the nanocone-arrays for a large area, which spontaneously provides the graded doping features for a selective emitter. This enables to lower the electrical contact resistance and enhances the carrier collection due to the high electric field distribution through a nanocone. Optically, the convex-shaped nanocones efficiently reduce light-reflection and the incident light is effectively focused into Si via nanocone structure, resulting in an extremely improved the carrier collection performances. This nanocone-arrayed selective emitter simultaneously satisfies optical and electrical improvement. We report the record high efficiency of $16.3 \%$ for the periodically nanoscale patterned emitter Si solar cell.

R esearchers have devoted to realize the high-performing photovoltaics $(\mathrm{PVs})^{1-5}$ to substitute the traditional and strong dependency on fossil energy resources. Generally, we may think two approaches to realize the high-efficient solar cells by considering electrical and optical aspects ${ }^{6-10}$. One is to drive more light energy into a light absorber, which can be achieved by reducing the reflection at a surface ${ }^{5-26}$. The other approach is to minimize the electrical loss, which enables to collect more number of photo-generated carriers with a minimum pay for recombination loss $s^{5,24,27,28}$.

Unfortunately, these two important issues are not compatible so far. This is caused by an unbalanced design in which, more weightage is given to the optical aspects. Gigantic progresses for the improvement of optical enhancements have been achieved in PV technologies ${ }^{6,18,29-32}$. According to researchers' consistent efforts, various types of entities, such as nanowires, nanostructures and microstructures have been applied for $\mathrm{PVs}^{5-8,12-26}$. We already achieved the near zero reflector for broadband wavelengths ${ }^{12,16,17,33}$. Meanwhile, the practical performances of the fancy solar cells $s^{5,8,12,14-24}$ are still much behind from the readily-achievable commercials $s^{3,31}$. Moreover, the fabrication of conceptual nanoscale solar cells is usually limited in large-scale applications.

We conceived a functional nanoscale design to take advantages over both the optical and electrical benefits. The biggest question in front of the photovoltaic researchers is "Is it possible to minimize an inevitable electrical loss and meanwhile, to maximize the optical enhancement in a solar cell?" The periodically shaped nanoscale emitter could be the possible solution to realize such a device. Periodically nanoscale grating structures can efficiently improve the optical path lengths with the substantial reduction of light reflection, therefore these structures provide a strong means for the optically enhanced $\mathrm{PVs}^{1,11,33,34}$. For an electrical aspect, a heavily-doped emitter provides a low-resistive path and therefore adds an advantage for carrier transportation. However, the higher dopant concentration easily degrades a carrier lifetime due to the Auger recombination ${ }^{6,35}$, while lightly-doped emitter is effective to relieve the serious recombination concern. At the same time, the dopant concentration in lightly-doped emitter causes a high contact resistance. These two conflicts limit the design scheme of an emitter. A selective-emitter has emerged to acquire the advantages of both the lightly- and heavily-doped emitters by 
sectioning the emitter regions for doping levels ${ }^{8,36,37}$. Conventional methods for the selective-emitter were achieved by using laser diffusion and metallization ${ }^{38}$. These methods were developed for a planar structure and therefore limited to apply for the nanoscale entities.

We herein, propose and demonstrate the nanoscale patterned $\mathrm{Si}$ solar cell, which have periodical selective-emitter arrays. This design was achieved by nanoimprint method which is suitable for largescale device fabrication. Commercial wet-etching process was applied to form the light-doped emitter region; meanwhile the heavily-doped emitter region was remained. As a result, we report the highest solar cell efficiency of $16.3 \%$ among the periodically nanoscale-patterned Si solar cells up to date. This result is substantially of improved efficiency compared to $13.7 \%$ of all-back-contact Si solar cells $s^{5}, 13.8 \%$ of compound InP nanowires solar cells ${ }^{21,22}$ or our previous nanolens solar cells ${ }^{1}$.

In this paper the following challenges and topics are addressed:

Large-scale fabrication of nanoscale Si patterns to have optical and electrical functions.

Optical enhancements to suppress light reflection at Si surface.

Electrical designs and performances of a selective-emitter solar cell.

Electrostatic analysis for $\mathrm{p} / \mathrm{n}$ junction and graded electric field distributions.

Traces of light propagation profiles along the nanoscale patterns.

\section{Results}

Wet-etching without a mask. To tailor the surface of the Si substrates, a wet-etching method was applied. Si (100) wafer has a degree of anisotropy and thus etching rates are different to crystal planes due to the difference of the Si bonding energy. The (111) planes work as an etching barrier, while the (100) planes are easily etched away. We used the wet-etching solution with a mixture of $\mathrm{NaOH}(2.5 \mathrm{wt} \%)$, isopropyl alcohol (5 wt\%), and de-ionized water (92.5 wt\%).

Figure 1a shows a SEM image of the pyramidal shaped Si substrate, having an angle of about $51^{\circ}$ between the (100) and (111) crystalline planes. The angle formation seems to be uniform through the pyramids, however, the sizes are varied by each pyramid (Fig. 1b) due to the different etching rate along the (100) plane even in a same solu$\operatorname{tion}^{39}$. Hereafter, this sample is referred as a textured Si sample.

Wet-etching with a mask for periodically patterned nanocone arrays. In order to fabricate periodic nanoscale structures, a largescale available nano-imprint method ${ }^{40}$ was applied to fabricate an etching mask. The fabrication sequences are presented in Figure 2a.
A polymethyl methacrylate (PMMA) layer was patterned as hole-arrays on a Si substrate, as shown in Figure 2b. After then, a thin $\mathrm{SiO}_{2}(20 \mathrm{~nm})$ layer was deposited on the hole-arrayed PMMA template. A lift-off process using an acetone solution was performed to remove the PMMA template and thus spontaneously removed the $\mathrm{SiO}_{2}$ layer, sitting on the PMMA layer. This procedure remained the inversely replicated $\mathrm{SiO}_{2}$ patterns on a Si substrate, as shown in Figure 2c. This $\mathrm{SiO}_{2}$ pattern works as an etching mask during the wet-etching process, which effectively relieves the different etching rate along the (100) planes, resulting in periodic nanoscale pyramids with uniform height.

We investigated to modulate the Si features by varying the time duration for the etching step. Si substrates showed grooved shapes (Fig. 2d) and nanoscale-pyramid arrays (Fig. 2e) for 2 min and $4 \mathrm{~min}$ etching time respectively. Due to the $\mathrm{SiO}_{2}$ etching mask, we can produce the periodic nanoscale pyramids, which has an angle of $54.7^{\circ}$ between the (100) plane and the sidewall.

Sharpening geometry is very efficient to reduce the light reflection, and thus for the enhanced solar cell performances. In order to achieve this structure, the optimum etching time is found to be as $8 \mathrm{~min}$. Uniformly patterned nanocones were fabricated to have a height of about $240 \mathrm{~nm}$ and a width of about $400 \mathrm{~nm}$ in a period of $500 \mathrm{~nm}$, as shown in Figure 2f. Hereafter, this structure is referred as a nanocone Si sample and used for a nanocone solar cell. As increasing the etching time, the Si may be over-etched to lose the etching shape. Figure $2 \mathrm{~g}$ is shown for an experimental demonstration for the over-etched Si structures.

Reflectance profiles. Photo-responses of a solar cell are strongly dependent on the utilization of the incident light. This can be realized by the reduction of the incoming light reflection on a surface, which spontaneously drives more photons into the lightactive semiconductor layer. Generally, we can realize the reduction of the incident light by means of two approaches. One way is to pattern the surface of the light-absorbing layer. The other approach is to adopt the graded refractive profiles. In order to achieve these two benefits, we designed a solar cell structure which is having a thin ITO film on the periodically patterned Si structures. For comparisons, three different types of Si structures were prepared as follows, a planar $\mathrm{Si}$, a textured $\mathrm{Si}$ and a nanocone $\mathrm{Si}$.

We clearly observed the structure dependent reflection tendencies from Figure 3a, showing the reflection profiles according to the $\mathrm{Si}$ structures. An average reflection value was obtained for the wavelength range of $300 \mathrm{~nm}-1100 \mathrm{~nm}$. A planar Si gave a high reflectance value of $34.42 \%$. Meanwhile, a textured $\mathrm{Si}$ effectively reduced the reflection value by $20.68 \%$. A significantly reduced reflection value of $8.55 \%$ was achieved from the nanocone Si.

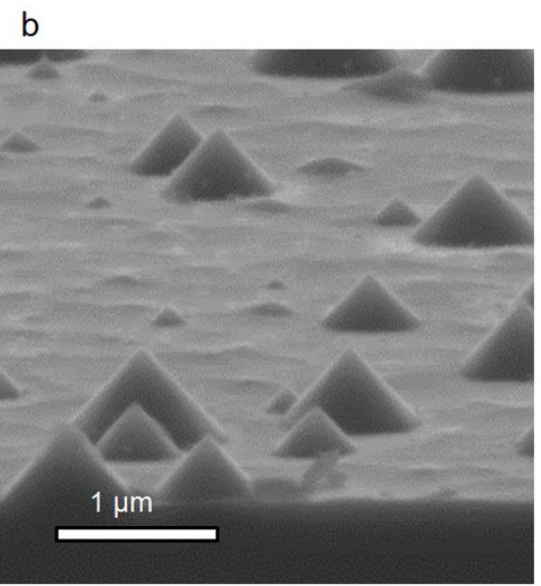

Figure $1 \mid$ SEM observation for a textured Si sample. Direct wet-etching process formed (a) a uniform pyramidal angle of $51^{\circ}$. However, the sizes are varied by each pyramid (b). 
a

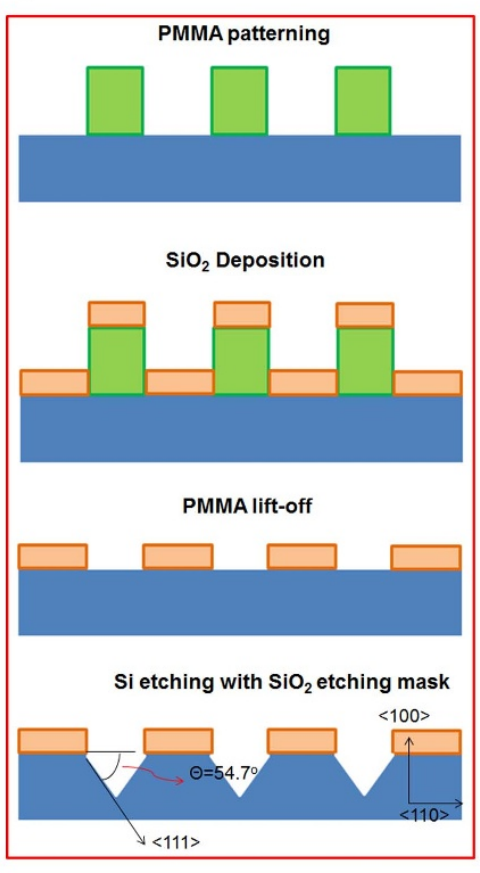

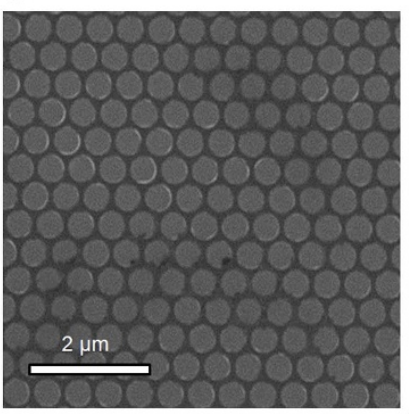

e

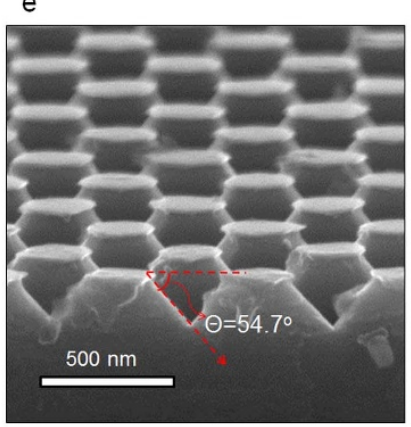

C

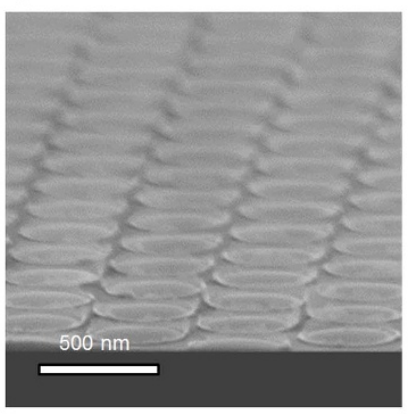

f

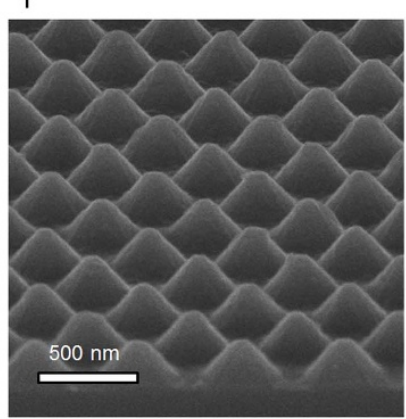

d

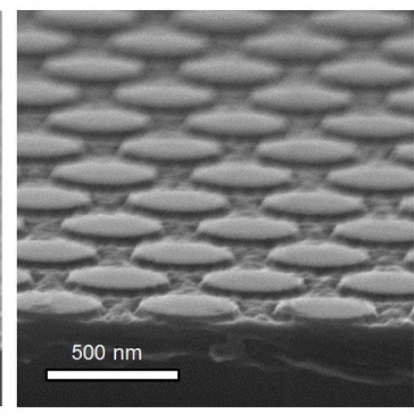

g

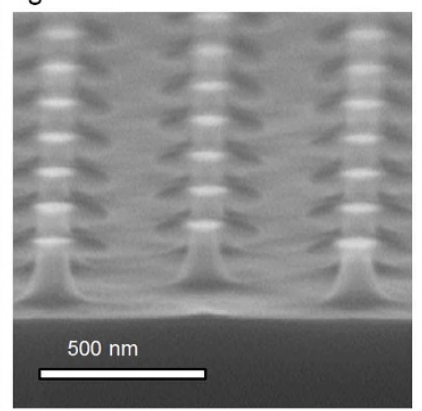

Figure $2 \mid$ Fabrication of periodic nanoscale Si structures. (a) Process flows. (b) PMMA patterns. (c) $\mathrm{SiO}_{2}$ patterns for etching-masks. Observation images of Si etched for (d) $2 \mathrm{~min}$, (e) $4 \mathrm{~min}$, (f) $8 \mathrm{~min}$, respectively. (g) A demonstration for over-etched Si structures.

A thin ITO film was coated on the different Si substrate samples for further improvement. As shown in Figure 3b, all three samples obviously presented the AR coating layer effect by using an ITO coating on $\mathrm{Si}$ structures. For a planar $\mathrm{Si}$ substrate, the reflection value was reduced to be $16.99 \%$. For a textured Si sample, the ITO coating significantly reduced the reflection value by $12.5 \%$. Moreover, the ITO coating on the periodic nanocone Si surface substantially suppressed the reflection value by $2.68 \%$ and also provided the near-zero reflection for broad wavelengths. Less than $1 \%$ reflection range was spread out throughout the wavelengths between $472 \mathrm{~nm}$ and $826 \mathrm{~nm}$ in nanocone Si structure. This remarkably low reflection is attributed to the graded refractive effects of ITO-coating along with the conical shaped Si structures. Moreover, the electrically conductive ITO coating layer also contributes to reduce contact resistance ${ }^{28}$, which has been discussed in the later part.

Figure $3 c$ is an SEM image of the Si nanocones with a top ITO layer. An ITO film actively works as an anti-reflection coating layer, due to its intermediate refractive index for the air-Si system. An optimum thickness of an ITO film was found to be $80 \mathrm{~nm}$, which is calculated by the quarter wavelength method $(d=\lambda / 4 n)$, in which $n$ is the refractive index of the $\mathrm{ITO}^{28}$.
Solar cell behaviors. To fabricate solar cell, the front and back silver metal electrodes were applied. The schematics of a nanocone solar cell are presented in Figure 4a. Three different types of solar cells were prepared with a planar $\mathrm{Si}$, a textured $\mathrm{Si}$ and a nanocone $\mathrm{Si}$. Each device were tailored for a large size of $3.2 \times 3.2 \mathrm{~cm}^{2}$, as shown in Figure $4 \mathrm{~b}$.

In order to investigate the diode characteristics, the I-V profiles for all devices were measured under dark condition. In general, all three samples provided good rectifying current flows (Fig. 4c). A planar Si device showed the lowest reverse saturation current $\left(I_{R S}\right)$, as expected. In comparison, a textured $\mathrm{Si}$ device and a nanocone $\mathrm{Si}$ device showed the increased $\mathrm{I}_{\mathrm{RS}}$ values. The reverse current amount is directly related to the defects along the Si surface, which is caused by the etching process ${ }^{20,24-26}$. Surface defects increase the leakage current and is usually proportional to the surficial enhancement. It is noteworthy to mention here that the nanocone $\mathrm{Si}$ device has a lower $I_{R S}$ value than that of the textured Si device. This shows that the random-texturing induces the severe defect problem, due to the non-directional etching surface.

Under forward bias, we can observe the relation between the surface enhancement and increased current. At a positive $0.8 \mathrm{~V}$, the a

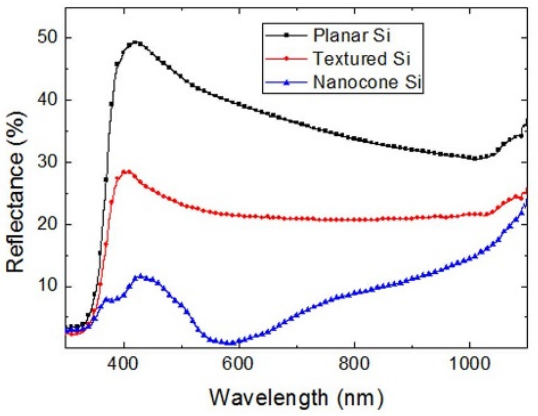

b

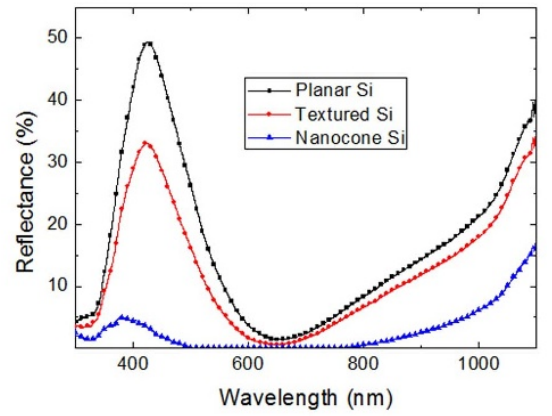

C

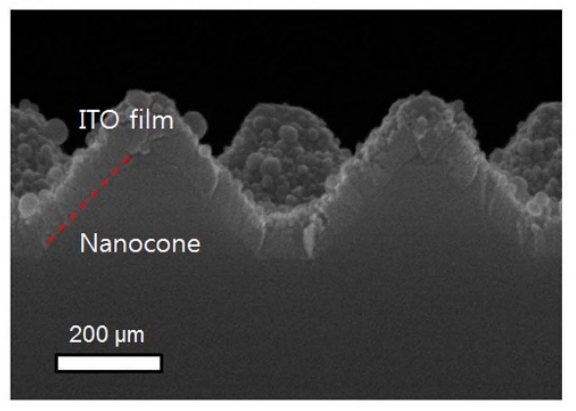

Figure $3 \mid$ Reflectance profiles. (a) Reflectance profiles of a planar, a textured, and a nanocone Si substrate. (b) Reflectance profiles after coating an ITO film over a planar, a textured, and a nanocone Si substrate. (c) SEM image of $80 \mathrm{~nm}$-thick ITO film coated nanocone Si arrays. 


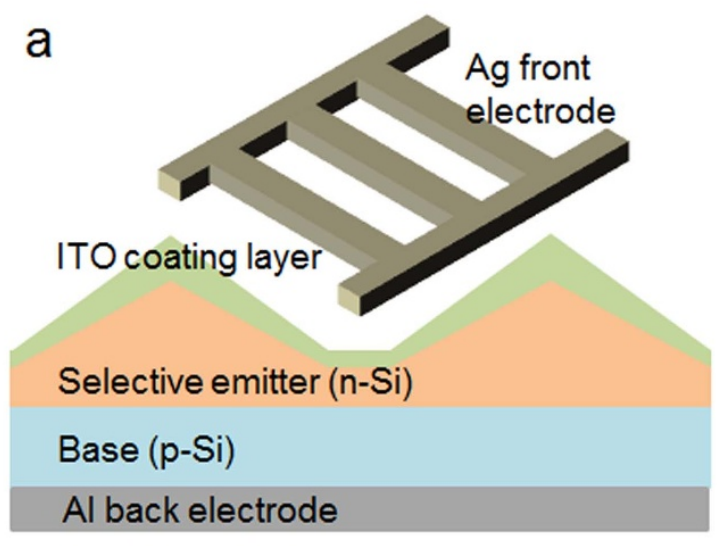

b

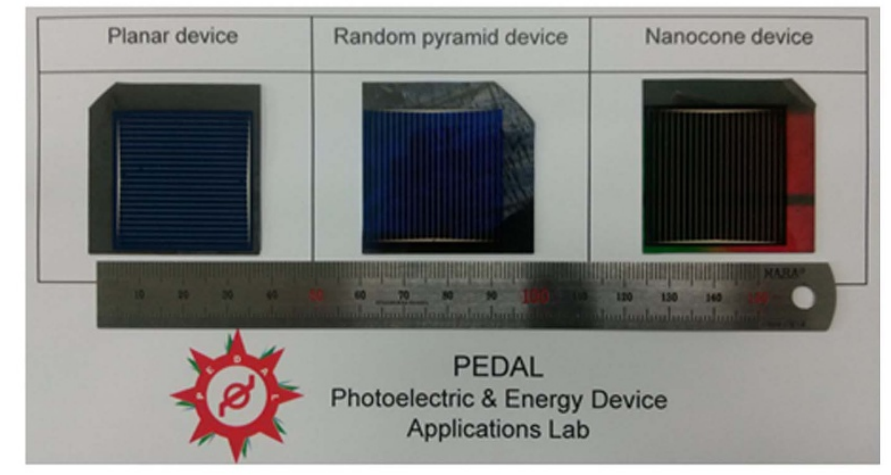

C
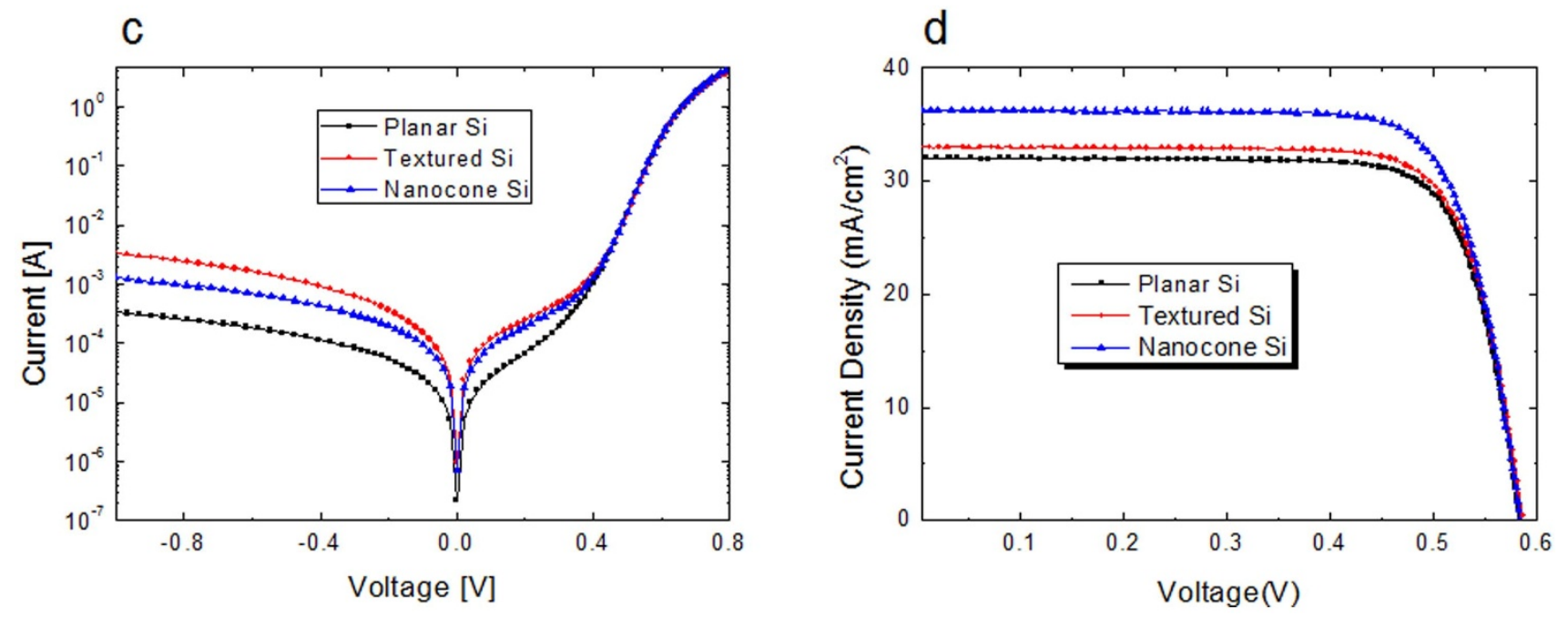

Figure 4 | Solar cell performances. (a) Schematic illustration of a nanocone solar cell. (b) Photo images of solar cell samples. I-V characteristics under (c) dark condition and (d) one-sun illumination.

nanocone device provided the highest forward current $\left(\mathrm{I}_{\mathrm{F}}\right)$ value of 4.12 A. Meanwhile, a random textured $\mathrm{Si}$ device showed a lower $\mathrm{I}_{\mathrm{F}}$ value than that of a planar device. To overlook the dark current tendencies, the rectifying ratios which are calculated from ratio of the current value at $+0.8 \mathrm{~V}$ to the current value at $-0.8 \mathrm{~V}$, were obtained and given in Table 1. A planar Si device has the highest rectifying ratio of 15152.5 , due mainly to the lowest $\mathrm{I}_{\mathrm{RS}}$ value. As next to the planar Si device, the nanocone Si device showed a rectifying ratio of 4367.3 which is higher than the 1499.5 of the random textured Si device.

In order to investigate a $\mathrm{p}-\mathrm{n}$ junction quality, the diode ideality factor $(n)$ was determined. The planar Si device provided $n$ value of 1.52 , showing a good $\mathrm{p}-\mathrm{n}$ junction quality. Etching-processed devices also showed good $n$ values of 1.57 for a nanocone device and 1.61 for a textured device. This implies that the recombination problem due to surface defects would be relieved by the surface enhancement. The $n$ value is related to a rate function of the voltage change over the current change as shown in the following equation:

$$
\mathrm{n}=\frac{q}{k T} \frac{\partial V}{\partial(\ln I)}
$$

where $\mathrm{kT}$ and $\mathrm{q}$ are the thermal energy and electron charge, respectively. The surface enhancement for the nanocone device effectively improves the current value, and thus renders a fair $n$ value.

In order to investigate the solar cell performances, the prepared devices were characterized using a simulator system (McScience$\mathrm{K} 3000)$ under one-sun $\left(100 \mathrm{~mW} / \mathrm{cm}^{2}\right)$ illumination in connection with a power meter (McScience-K101). The periodic nanocone solar cell provided a substantially improved conversion efficiency of $16.3 \%$ (Supplementary section 'Calculation of solar cell efficiency'), compared to $14.5 \%$ of a planar solar cell or $15.0 \%$ of a texture solar cell. This nanocone solar cell efficiency $(16.3 \%)$ is considered to be the highest efficiency value among the periodic nanoscale-patterned $\mathrm{Si}$ solar cells up to date ${ }^{22}$. An open circuit voltage $\left(V_{o c}\right)$ is an important factor to accomplish a high efficient nanoscale solar cell. In general, the nanostructured solar cells tend to degrade $V_{o c}$ values by the trap density in a space charge region $(S C R)^{9}$. From the results, all three samples have a relatively similar open-circuit voltage values (583$586 \mathrm{mV}$ ). There was no severe $V_{o c}$ degradation observed in the nanocone $\mathrm{Si}$ solar cell and this result is different from previous reports ${ }^{15,17,23}$. This result clearly demonstrates a fair formation of the SCR of the nanocone solar cell.

The enhanced efficiency of the nanocone solar cell is mostly attributed to the improved current value along with the periodic nanoscale Si feature. The short-circuit current density $\left(J_{s c}\right)$ of the nanocone solar cell was achieved to be $36.25 \mathrm{~mA} / \mathrm{cm}^{2}$. This is a significantly improved current value from $32.05 \mathrm{~mA} / \mathrm{cm}^{2}$ of the planar device. The current ratio (light-induced current/reverse saturation current) also directly controls the $V_{o c}$ value, according to the following relation:

$$
V_{o c}=\frac{k T}{q} \ln \frac{I_{\text {light }}}{I_{R S}}+1
$$

where $I_{\text {light }}$ is the light-induced current. For a nanocone solar cell, the defect-induced effect (increased $I_{R S}$ value) was sufficiently relieved by 
Table 1 | Device performances

\begin{tabular}{lccc} 
& Planar Si & Texture Si & Nanocone Si \\
\hline$I_{R S}(m A)$ & 0.3 & 2.4 & 0.9 \\
$I_{F}(A)$ & 3.79 & 3.58 & 4.12 \\
Ideality factor & 1.52 & 1.61 & 1.57 \\
Rectifying ratio & 15152.5 & 1499.5 & 4367.3 \\
Surface enhancement & $100 \%$ & $\mathrm{~N} / \mathrm{A}$ & $133.9 \%$ \\
$\mathrm{~V}_{\text {oc }}(\mathrm{mV})$ & 584 & 586 & 583 \\
$\mathrm{~J}_{\text {sc }}\left(\mathrm{mA} / \mathrm{cm}^{2}\right)$ & 32.05 & 32.86 & 36.25 \\
Cell efficiency $(\%)$ & 14.5 & 15.0 & 16.3 \\
\hline
\end{tabular}

the significantly increased $I_{\text {light }}$. Meanwhile, no significant current improvement was achieved for the texture solar cell $(32.86 \mathrm{~mA} /$ $\mathrm{cm}^{2}$ ) due to the smaller surface than that of a nanocone Si device.

In order to investigate the current behaviors, the surface enhancements of the periodic nanocone structures have been calculated. Nanocones are periodically arrayed with a period of about $500 \mathrm{~nm}$ on the entire device surface. A single nanocone has a width of $400 \mathrm{~nm}$ and a height of $240 \mathrm{~nm}$. By considering a planar surface $(100 \%)$ as a reference, the nanocone structure has the surface area by $133.9 \%$ in a unit cell and thus increases the light-reactive Si region. This also simultaneously enlarges an interface between Si and ITO. Due to the increased ITO surface along the nanocone-arrays, the nanocone device provided a low series resistance value of $1.546 \Omega$ $\mathrm{cm}^{2}$, which is much smaller than the $5 \Omega \mathrm{cm}^{2}$ of nanowire surface or close to a theoretical value ${ }^{14}$ of $1.5 \Omega \mathrm{cm}^{2}$.
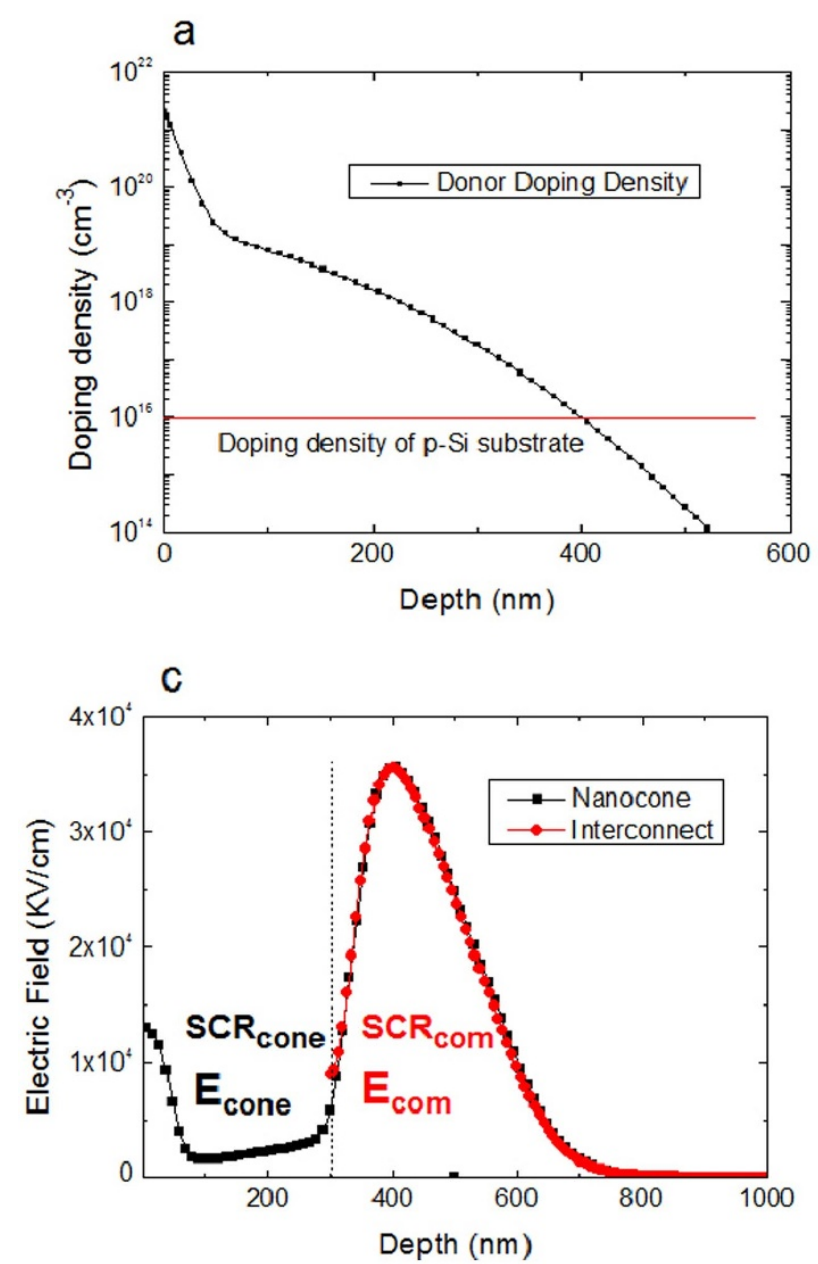

In an electrical aspect, an ITO layer supports carrier transport from a Si to a metal electrode. As a result, the enhanced surface area by nanocone-arrays improved the light-induced current $\left(I_{\text {light }}\right)$ and diode forward current $\left(I_{F}\right)$, as well.

Analyses for solar cells. A distinctive feature of the nanocone device is to have a selective-emitter. The etching process spontaneously tailors the emitter region to have convex nanocone-arrays, which is connected by an interconnect region. The interconnect region is located at a depth of $240 \mathrm{~nm}$ from the top surface. The doping profiles were analyzed using secondary ion mass spectroscopy investigation has been performed, as shown in Figure 5a. We obtained the donor concentration $\left(N_{d}\right)$ of about $10^{18} / \mathrm{cm}^{3}$ for the interconnect emitter region. Considering the acceptor concentration of the $\mathrm{p}$-Si substrate $\left(10^{16} / \mathrm{cm}^{3}\right)$, the $\mathrm{p} / \mathrm{n}$ junction between the $\mathrm{n}$-Si emitter side and the $\mathrm{p}$-Si base formed a space charge region $(S C R)$. This is established by major carrier diffusion mechanism. Electrons move from $\mathrm{n}-\mathrm{Si}$ to $\mathrm{p}-\mathrm{Si}$ and holes move to the opposite direction. Electrons and holes, fixed in SCR, establish an electric field, which is a driving force to collect the photo-generated carriers.

A main advantage of the periodic nanocone device is to render the graded doping profile through each nanocone structure, as depicted in Figure 5b. The peak doping concentration $\left(\mathrm{N}_{\mathrm{d}}=10^{21} / \mathrm{cm}^{3}\right)$ exists at a top of a nanocone and the doping concentration is gradually decreased with increasing distance from the top surface. This graded doping concentration in the nanocone geometry gives an additional electric field, and which correspondingly widens SCR.
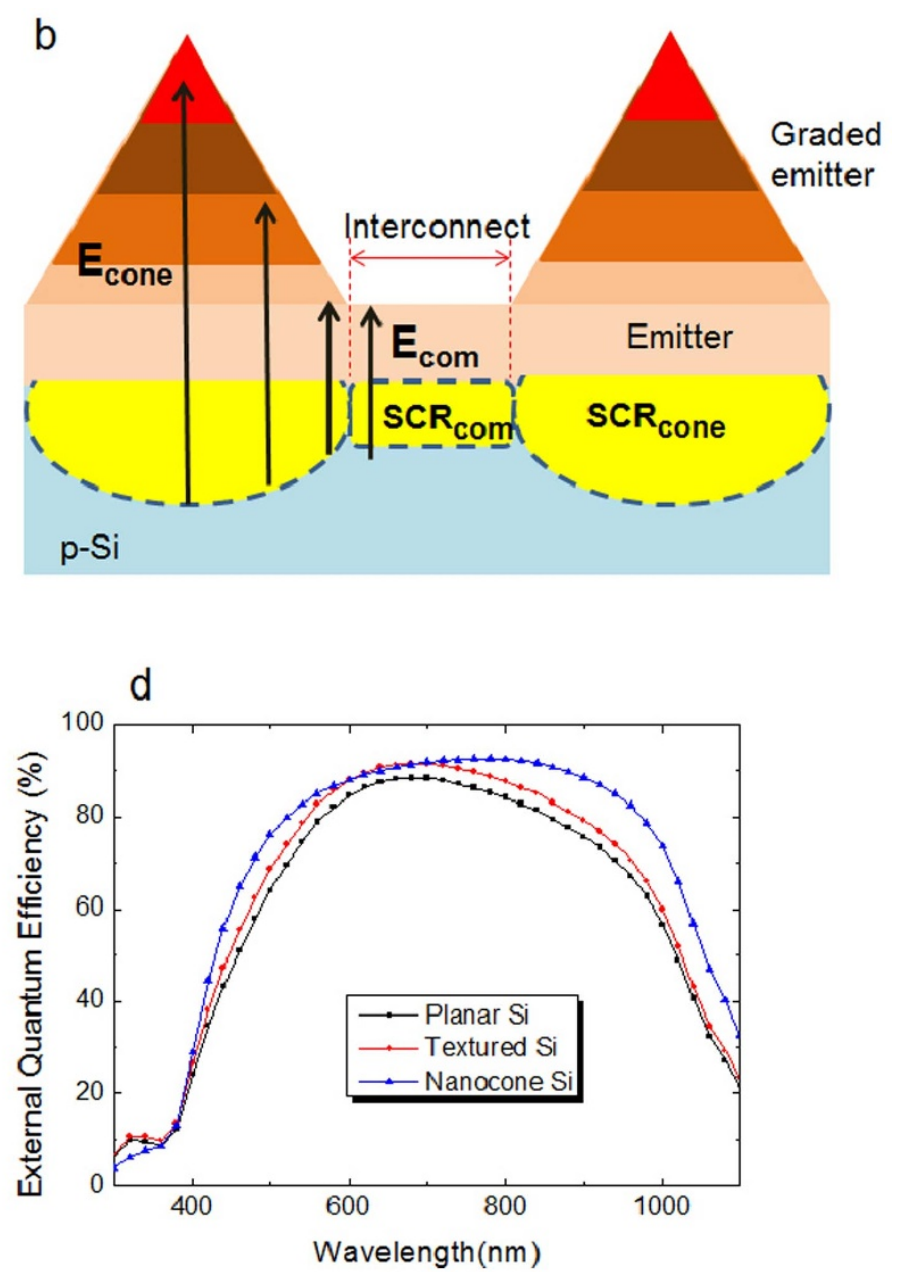

Figure 5 Selective emitter analyses. (a) Doping profiles for n-Si and p-Si. (b) Schematics of an emitter doping-graded nanocone solar cell. (c) Electric field distribution profiles. (d) Quantum efficiencies for solar cells. 
The electrostatic profiles of the different shaped $\mathrm{p} / \mathrm{n}$ junction were analyzed using PC1D program, as presented in Figure $5 \mathrm{c}$. For the interconnect region, the electric field is distributed along the SCR, showing the peak value of $37 \mathrm{kV} / \mathrm{cm}$ at the center of $S C R$. This is caused by a common $E\left(E_{c o m}\right)$, co-existed both for a nanocone and an interconnect regions due to the common n-doping level $\left(\mathrm{N}_{\mathrm{d}}=10^{18}\right.$ / $\left.\mathrm{cm}^{3}\right)$. We denote the $S C R$ formed by $E_{\text {com }}$ as $S C R_{\text {com }}$, in Figure $5(\mathrm{~b}, \mathrm{c})$. Different from the interconnect region, the nanocone-shaped emitter has the graded doping levels, which widens the SCR space, as denoted $S C R_{\text {cone }}$. This was induced by an additional $E$ distribution, which is denoted as $E_{\text {cone }}$, in Figure $5 \mathrm{c}$.

This doping-level graded emitter, having nanocone interconnected arrays, is spontaneously providing the feature of a selective emitter. The heavily-doped region contributes the carrier transport due to the enhanced $E$, which also provides a low-resistive path for photo-generated carriers. Meanwhile, the interconnect region has a benefit to reduce the recombination loss due to its low dopants concentration.

To examine the carrier collection performance, the external quantum efficiencies (EQE) were measured for all samples. The nanocone solar cell showed the remarkably enhanced carrier collection efficiencies for broad wavelengths compared to those of the texture or the planar devices, as shown in EQE (Fig. 5d). Precisely, the quantum efficiency of more than $85 \%$ is achieved by nanocone solar cell at a broad wavelength range of 600-900 nm which covers the important region for $\mathrm{Si}$ based solar cell devices. This clearly demonstrates that the nanocone-shaped selective emitter is very effective to collect the photo-generated carriers. It is worthy to note that QE improves enormously at longer wavelengths. At a wavelength of $1100 \mathrm{~nm}$, the nanocone solar cell improved QE value by $50.6 \%$ from that of a planar solar cell (Supplementary section 'Relative EQE). For a wavelength of $600 \mathrm{~nm}$, a relatively low QE improvement $(\sim 4 \%)$ was obtained. This is caused by the high QE value $(84.62 \%)$ of a planar device inherent from a low reflectance at the corresponding wavelength (Fig. $3 \mathrm{~b}$ ). The nanocone structure is also effective for short wavelengths. At a wavelength of $440 \mathrm{~nm}$, the nanocone device showed a $29.3 \%$ enhanced QE value from that of a planar device. However, we observed the degraded QE performances for $\lambda<360 \mathrm{~nm}$. This is attributed to the defects-induced recombination effect along the enhanced $\mathrm{Si}$ surface, resulting in the degraded QE values at very short wavelengths. We can also consider the Auger recombination effect for a heavily-doped emitter region $\left(\mathrm{N}_{\mathrm{d}}>10^{20} / \mathrm{cm}^{3}\right)^{6,35}$. By considering photon absorption for $\lambda<$ $400 \mathrm{~nm}, \mathrm{Si}$ has a very short absorption length less than $100 \mathrm{~nm}$. This causes a serious Auger recombination loss for the short wavelength photons. We can observe that the texture Si device showed the improved QE values at short wavelengths, due to its relatively light doping concentration in an emitter.

Light behaviors by the nanocone structure. Besides the electrical features, it is also critical to investigate the light propagation of incident light behaviors through the nanocone geometry. To understand the optical characteristics of the nanocone solar cells, Maxwell's equations were numerically solved using the finitedifference time-domain (FDTD) simulations (Lumerical FDTD Solutions). The devices were modeled with unit cells and the proper boundary conditions. The light-induced electric field $\left(E_{\text {light }}\right)$ intensity distributions and reflectance spectra were obtained under illumination of linearly polarized light.

Figure 6 shows the electric field distributions for a nanocone structure $(a-d)$ and a planar structure $(e-f)$, at different wavelengths $(\lambda)$. For a planar structure, the incident light propagates as a form of plane wave. Meanwhile, the incoming light is significantly concentrated near the nanocone surface. The light propagation is modulated by passing the nanocone structure and $E_{\text {light }}$ distribution profiles are highly dependent on the light wavelengths.

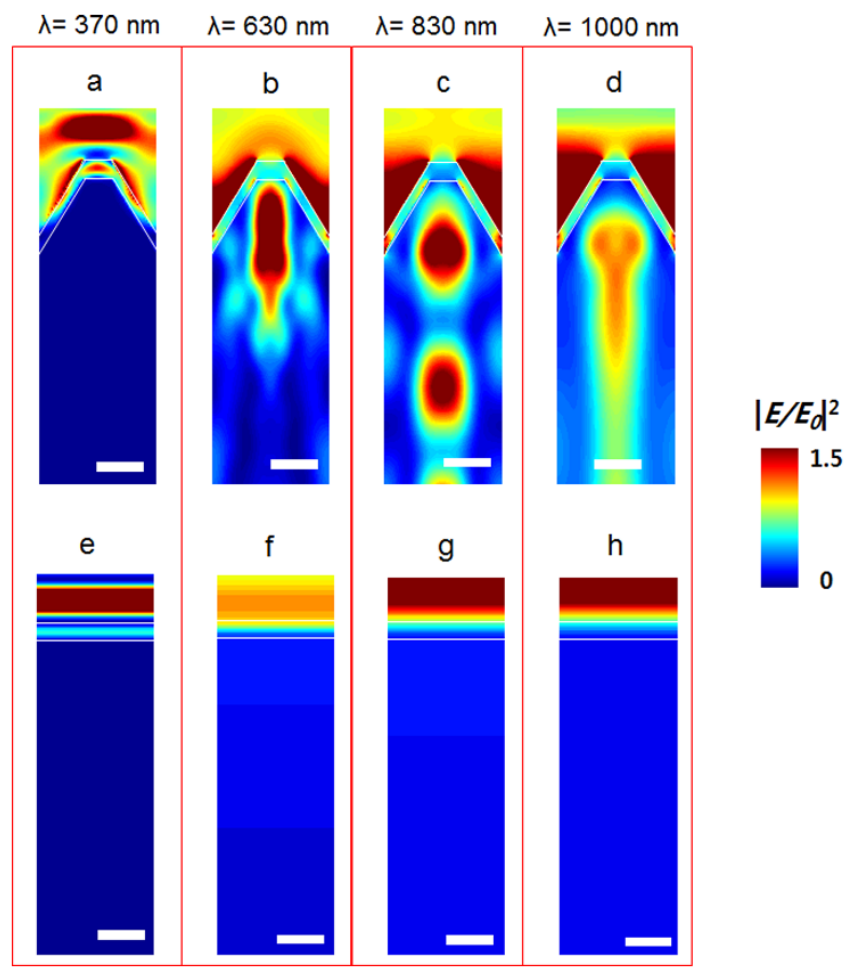

Figure 6 | FDTD simulations. Light-induced electric field distributions for a different wavelength at $370 \mathrm{~nm}, 630 \mathrm{~nm}, 830 \mathrm{~nm}$, and $1000 \mathrm{~nm}$ for a nanocone $\mathrm{Si}$ structure (a-d) and a planar $\mathrm{Si}(\mathrm{e}-\mathrm{f})$. The scale bar is $100 \mathrm{~nm}$.

At $\lambda=370 \mathrm{~nm}$, the largest field intensity appears at the air/ITO interface and the intensity strongly decays in the ITO layer. As ITO layer is an electrical conductor, it contributes the photo-generated carrier collections. In contrast, the ITO film causes a free carrier loss, without creating the electron-hole pairs for short wavelengths ${ }^{41,42}$. This suggests that very limited incident photons can reach the $\mathrm{Si}$ active region in the solar cell, resulting in negligibly small QE at $\lambda<400 \mathrm{~nm}$ (Fig. 6a).

At $\lambda=630 \mathrm{~nm}$, very strong light intensity is seen in the center of a nanocone region (Fig. $6 \mathrm{~b}$ ). Such field pattern could be attributed to the excitation of the resonant guided modes and the antireflection effects due to the nanocone patterns.

For long wavelengths $(\lambda=830$ and $1000 \mathrm{~nm})$, the nanocone structure generated strong $E_{\text {light }}$ and which propagated into a deep Si region, along the center line of a nanocone (Fig. $6 c$ and d). Light trapping of long wavelengths $(\lambda>800 \mathrm{~nm})$ is very important for $\mathrm{Si}$ solar cell, which is directly related to improved performance of the solar cell using the low photon-energy effectively ${ }^{41,43}$. The nanocone shaped $\mathrm{Si}$ device is effective to focus the photons of longer wavelengths and prominently improved QE values for longer wavelengths.

In summary, we have reported the record high efficiency of $16.3 \%$ among the nanoscale patterned Si solar cells. Periodically patterned Si nanocones were achieved by a large area applicable nanoimprint method. $\mathrm{SiO}_{2}$-masks were arrayed on a planar $\mathrm{Si}$ substrate and were effective to provide the periodic Si nanocone by using a wet-etching process. The nanocone-shaped surface is enormously reduces the reflection of incident light and also drives more photons into the $\mathrm{Si}$ material. Beside these optical benefits, the nanocone-embedded emitter is electrically advantageous to have graded doping profiles via nano entities. According to the high $\mathrm{n}$-doping concentration, an enhanced electric field is formed through the nanocone structure. This enhanced electric field existing in the nanocone regime is beneficial to collect the photo-generated carriers and also provides a low resistive path. Meanwhile, the nanocone interconnect region has a 
relatively low dopants, and thus which significantly relieves the recombination loss of photo-generated carrier. We demonstrated the high-performing nanoscale patterned Si solar cell, which realizes the optical benefits into the real electrical improvement. We suggest that this type of nanoscale solar cell will achieve further enhanced performance by controlling the doping profiles of an emitter and scaling of nanostructures for light propagation.

\section{Methods}

PMMA patterning. Large-scale applicable nanoimprint method applied to nanoscale patterned PMMA layer. A spin coating method was used to deposit $250 \mathrm{~nm}$-thick PMMA layer on a 4-inch Si wafer, at $1000 \mathrm{rpm}$ for $60 \mathrm{~s}$. After then, UV-curable resin was coated onto the PMMA layer. To make nanoscale patterns in the UV-resin, UV imprinting process was performed by using an NIL-8 imprinter (Obducat, Sweden) under exposing $365 \mathrm{~nm}-\mathrm{UV}$ light $\left(25 \mathrm{~mW} / \mathrm{cm}^{2}\right)$. $\mathrm{O}_{2}$ reactive ion etching $\left(\mathrm{O}_{2}\right.$-RIE) process was followed to tailor the UV-resin-sitting PMMA layer. During this etching process, the nanoscale-patterned UV-resin layer works as an etching mask to transmit its shape to nanoscale hole-arrayed PMMA patterns. A field emission scanning electron microscope (FESEM, FEI Sirion) was employed to observe the $\mathrm{Si}$ structures. The doping concentration profile was investigated by secondary ion mass spectroscopy (SIMS, Cameca, magnetic sector ims7f).

Junction formation. The $\mathrm{p} / \mathrm{n}$ junction was obtained by a thermal doping method. We used a Czochralski (CZ) grown 4-inch p-type (100) Si wafer, having a doping level of $10^{16} / \mathrm{cm}^{3}$. This $\mathrm{p}$-Si wafer loaded in a doping furnace and the $\mathrm{n}$-doping process was performed. During the doping process, a temperature was maintained at $800^{\circ} \mathrm{C}$. Phosphoryl oxychloride $\left(\mathrm{POCl}_{3}\right)$ was flown as a doping agency with the mixed $\mathrm{N}_{2}$ and $\mathrm{O}_{2}$ gases for $30 \mathrm{~min}$. A buffered hydrofluoric acid (5\% HF) solution was used to remove phosphosilicate glass (PSG).

ITO layer coating. In order to deposit ITO layer on a Si substrate, a DC magnetron sputtering system (SNTEK, Korea) was used. During the sputtering, a DC power source $\left(3.70 \mathrm{~W} / \mathrm{cm}^{2}\right)$ was applied to a 4 -inch ITO target $\left(\operatorname{In}_{2} \mathrm{O}_{2}\right.$ containing $10 \mathrm{wt} \%$ $\mathrm{SnO}_{2}$ ). This was performed under $\mathrm{Ar}$ atmospheric condition at a temperature of $300^{\circ} \mathrm{C}$.

Electrode formation. The front and back metal electrodes were fabricated for solar cells. A back electrode was achieved by a printing method. Al paste was printed on the back Si substrate and a firing process was performed to establish a back surface field. For a front electrode, a grid pattern was employed by a screen printing of Ag paste on the ITO film, followed by dry process at $150^{\circ} \mathrm{C}$ for $5 \mathrm{~min}$.

One-sun simulation. A simulator system (McScience-K3000, Korea) was employed to measure solar cell performances. A photovoltaic power meter (McScience-K101) was used to monitor the I-V characteristics under one sun $\left(100 \mathrm{~mW} / \mathrm{cm}^{2}\right)$ illumination. Carrier collection efficiencies of solar cells were profiled by using a quantum efficiency measurement system (McScience-K3100).

1. Yun, J. H. et al. Incident light adjustable solar cell by periodic nanolens architecture. Sci. Rep. 4, 6879 (2014).

2. Ye, X. et al. 18.45\%-Efficient Multi-Crystalline Silicon Solar Cells with Novel Nanoscale Pseudo-Pyramid Texture. Adv. Funct. Mater. 24, 6708-6716 (2014).

3. De Wolf, S., Descoeudres, A., Holman, Z. C. \& Ballif, C. High-efficiency Silicon Heterojunction Solar Cells: A Review. green 2, 7-24 (2012).

4. Priolo, F., Gregorkiewicz, T., Galli, M. \& Krauss, T. F. Silicon nanostructures for photonics and photovoltaics. Nat. Nanotechnol. 9, 19-32 (2014).

5. Jeong, S., McGehee, M. D. \& Cui, Y. All-back-contact ultra-thin silicon nanocone solar cells with 13.7\% power conversion efficiency. Nat. Commun. 4, 1-7 (2013).

6. Oh, J., Yuan, H.-C. \& Branz, H. M. An 18.2\%-efficient black-silicon solar cell achieved through control of carrier recombination in nanostructures. Nat. Nanotechnol. 7, 743-748 (2012).

7. Chen, T.-G. et al. Characteristics of large-scale nanohole arrays for thin-silicon photovoltaics. Prog. Photovoltaics Res. Appl. 22, 452-461 (2014).

8. Um, H.-D. et al. Incorporation of a self-aligned selective emitter to realize highly efficient (12.8\%) Si nanowire solar cells. Nanoscale 6, 5193-5199 (2014).

9. Kim, H. et al. Effect of the short collection length in silicon microscale wire solar cells. Appl. Phys. Lett. 102, 193904 (2013).

10. Kim, J. et al. Surface-concentrated light and efficient carrier collection in microhole-patterned Si solar cells. Opt. Express 21, A607-A615 (2013).

11. Battaglia, C. et al. Light Trapping in Solar Cells: Can Periodic Beat Random? ACS Nano 6, 2790-2797 (2012).

12. Liu, Y. et al. Nanostructure formation and passivation of large-area black silicon for solar cell applications. Small 8, 1392-7 (2012).

13. Alaeian, H., Atre, A. C. \& Dionne, J. A. Optimized light absorption in Si wire array solar cells. J. Opt. 14, 024006 (2012).

14. Perraud, S. et al. Full process for integrating silicon nanowire arrays into solar cells. Sol. Energy Mater. Sol. Cells 93, 1568-1571 (2009).

15. Adachi, M. M., Anantram, M. P. \& Karim, K. S. Core-shell silicon nanowire solar cells. Sci. Rep. 3, 1546 (2013)
16. Guobin, J. et al. Silicon Nanowire Solar Cells With Radial p-n Heterojunction on Crystalline Silicon Thin Films: Light Trapping Properties. IEEE J. Photovoltaics 4, 28-32 (2014).

17. He, L., Lai, D., Wang, H., Jiang, C. \& Rusli. High-efficiency si/polymer hybrid solar cells based on synergistic surface texturing of Si nanowires on pyramids. Small $\mathbf{8}$, 1664-8 (2012).

18. Ferry, V. E., Polman, A. \& Atwater, H. A. Modeling Light Trapping in Nanostructured Solar Cells. ACS Nano 5, 10055-10064 (2011).

19. Jung, Y., Li, X., Rajan, N. K., Taylor, A. D. \& Reed, M. A. Record high efficiency single-walled carbon nanotube/silicon p-n junction solar cells. Nano. Lett. 13, 95-9 (2013).

20. Seo, K. et al. Si Microwire Solar Cells: Improved Efficiency with a Conformal SiO2 Layer. ACS Nano 7, 5539-5545 (2013).

21. Tan, H., Santbergen, R., Smets, A. H. \& Zeman, M. Plasmonic light trapping in thin-film silicon solar cells with improved self-assembled silver nanoparticles. Nano Lett. 12, 4070-6 (2012).

22. Krogstrup, P. et al. Single-nanowire solar cells beyond the Shockley-Queisser limit. Nat. Photonics 7, 306-310 (2013).

23. Gharghi, M., Fathi, E., Kante, B., Sivoththaman, S. \& Zhang, X. Heterojunction silicon microwire solar cells. Nano Lett. 12, 6278-82 (2012).

24. Lee, E. et al. Comparative experimental and simulative investigations of radial $\mathrm{p}-\mathrm{n}$ junction Si microwire array solar cells. Sol. Energy Mater. Sol. Cells 103, 93-97 (2012).

25. Ferry, V. E. et al. Optimized spatial correlations for broadband light trapping nanopatterns in high efficiency ultrathin film a-Si:H solar cells. Nano Lett. 11, 4239-45 (2011)

26. Wallentin, J. et al. InP nanowire array solar cells achieving $13.8 \%$ efficiency by exceeding the ray optics limit. Science 339, 1057-60 (2013).

27. Vinod, P., Chakravarty, B., Lal, M., Kumar, R. \& Singh, S. A novel method for the determination of the front contact resistance in large area screen printed silicon solar cells. Semicond. Sci. Technol. 15, 286 (2000).

28. Kim, M. et al. Optimization of transparent conductor-embedding front electrodes for efficient light management. Curr. Appl. Phys. 13, 808-813 (2013).

29. Yu, Z., Raman, A. \& Fan, S. Fundamental limit of nanophotonic light trapping in solar cells. Proc. Natl. Acad. Sci. U.S. A. 107, 17491-6 (2010).

30. Li, Y. et al. Novel silicon nanohemisphere-array solar cells with enhanced performance. Small 7, 3138-43 (2011)

31. Mavrokefalos, A., Han, S. E., Yerci, S., Branham, M. S. \& Chen, G. Efficient light trapping in inverted nanopyramid thin crystalline silicon membranes for solar cell applications. Nano Lett. 12, 2792-6 (2012).

32. Zhu, J., Hsu, C. M., Yu, Z., Fan, S. \& Cui, Y. Nanodome solar cells with efficient light management and self-cleaning. Nano Lett. 10, 1979-84 (2010).

33. Yoo, J., Yu, G. \& Yi, J. Black surface structures for crystalline silicon solar cells. Mater. Sci. Eng. B 159-160, 333-337 (2009).

34. Han, S. E. \& Chen, G. Optical absorption enhancement in silicon nanohole arrays for solar photovoltaics. Nano Lett. 10, 1012-5 (2010).

35. Diedenhofen, S. L., Janssen, O. T. A., Grzela, G., Bakkers, E. P. A. M. \& Gómez Rivas, J. Strong Geometrical Dependence of the Absorption of Light in Arrays of Semiconductor Nanowires. ACS Nano 5, 2316-2323 (2011).

36. Green, M. A. Limits on the open-circuit voltage and efficiency of silicon solar cells imposed by intrinsic Auger processes. IEEE Trans. Electron Devices, 31, 671-678 (1984).

37. Moon, I. et al. Selective emitter using porous silicon for crystalline silicon solar cells. Sol. Energy Mater. Sol. Cells 93, 846-850 (2009).

38. Zhu, L. Q. et al. Improving the efficiency of crystalline silicon solar cells by an intersected selective laser doping. Sol. Energy Mater. Sol. Cells 95, 3347-3351 (2011).

39. Park, H.-H. et al. Wafer-scale surface roughening for enhanced light extraction of high power AlGaInP-based light-emitting diodes. Opt. Express 22, A723 (2014).

40. Kim, H. et al. Two-Step Texture Process for High-Efficiency Crystalline Silicon Solar Cell Applications. J. Nanosci. Nanotechnol. 13, 7916-7919 (2013).

41. Holman, Z. C. et al. Infrared light management in high-efficiency silicon heterojunction and rear-passivated solar cells. J. Appl. Phys. 113, 013107 (2013).

42. Holman, Z. C. et al. Current Losses at the Front of Silicon Heterojunction Solar Cells. IEEE J. Photovolatics 2, 7-15 (2012)

43. Wang, W., Wu, S., Reinhardt, K., Lu, Y. \& Chen, S. Broadband Light Absorption Enhancement in Thin-Film Silicon Solar Cells. Nano Lett. 10, 2012-2018 (2010).

\section{Acknowledgments}

The authors acknowledge the financial support of the Korea Institute of Energy Technology Evaluation and Planning, in a grant funded by the Ministry of Knowledge and Economy (KETEP-20133030011000)

\section{Author contributions}

J.K. conceived this research. J.H.Y. designed the selective emitter solar cell devices. H.K. fabricated solar cells. Y.C. performed FDTD simulation. H.H.P. fabricated PMMA and $\mathrm{SiO}_{2}$ patterns. M.M.D.K. analyzed device performances. J.Y. and W.A.A. contributed to design solar cells. J.Y. supervised H.K., D.W.K. supervised Y.C. All the authors contributed to prepare this manuscript. 


\section{Additional information}

Supplementary information accompanies this paper at http://www.nature.com/ scientificreports

Competing financial interests: The authors declare no competing financial interests.

How to cite this article: Kim, J. et al. Transparent conductor-embedding nanocones for selective emitters: optical and electrical improvements of Si solar cells. Sci. Rep. 5, 9256; DOI:10.1038/srep09256 (2015).

This work is licensed under a Creative Commons Attribution 4.0 International License. The images or other third party material in this article are included in the article's Creative Commons license, unless indicated otherwise in the credit line; if the material is not included under the Creative Commons license, users will need to obtain permission from the license holder in order to reproduce the material. To view a copy of this license, visit http://creativecommons.org/licenses/by/4.0/ 